Proceeding Series of the Brazilian Society of Computational and Applied Mathematics

\title{
Analysis and comparison of two oscillatory feedback control schemes for stabilizing equilibrium points.
}

\section{Verónica Estela Pastor ${ }^{1}$}

Departamento de Matemática, Facultad de Ingeniería, UBA, Buenos Aires, Argentina Graciela Adriana González ${ }^{2}$

Departamento de Matemática, Facultad de Ingeniería, UBA, Buenos Aires, Argentina, Consejo Nacional de Investigaciones Científicas y Técnicas, Argentina

\begin{abstract}
Two methods based on oscillatory delayed feedback control schemes for the continuous time case are dealt with. For both of them, stabilization of an equilibrium point in the general non-linear scalar case is rigorously proven. Additionally, their control performance and stability parameters region are respectively studied.
\end{abstract}

Keywords. oscillating feedback control, delay, stability region, control performance, rate of convergence.

\section{Introduction}

It is well known that delayed feedback control was originally proposed by Pyragas in [3]. Its most important feature is that it does not require the exact location of the unstable periodic orbit (UPO) to be stabilized. It makes use of a control signal obtained from the difference between the current state of the system and the state of the system delayed by one period of the UPO. An extended version, proposed in [7] results more effective for stabilizing great period orbits and high-unstable equilibrium points [4].

It is pointed out in [4] that equilibrium point stabilization is achieved by this technique if the degrees of freedom is greater or equal to two and only for certain kind of instabilities. Moreover, it is rigorously proved in [2] that if the linear variational equation about the target UPO has an old number of real characteristic multipliers greater than unity, the UPO cannot be stabilized by any one of these methods. This limitation has been anticipated by [6] inspired in Giona's proof for systems in which the discrete version is applied via Poincaré maps. To overcome this drawback, a new algorithm is designed in [5] by artificially enlarging a set of real multipliers greater than unity to an even number.

Another proposal for improving the delayed feedback limitation is based on an oscillatory delayed feedback [6]. The key of this strategy is to avoid a too rapid decay of the control

\footnotetext{
${ }^{1}$ vpastor@fi.uba.ar

2 ggonzal@fi.uba.ar
} 
magnitude and this could be achieved by applying feedback control only periodically. As stated in [5], the related stabilizing result is not clear. In spite of this, it was re-formulated in [1] for the discrete-time case where its validity for stabilizing periodic orbits of a wide class of maps is fully argued.

In the second part of the paper [6], the idea of an oscillating delayed feedback control is translated to the differential equations. Stabilization can not be achieved by an oscillating perturbation term based on the difference between current state and a delayed state. Instead, an oscillatory velocity term is proposed although it is limited to stabilization of equilibrium point of a scalar linear differential equation, with a rather uncompleted proof. In this work, stabilization of equilibrium points by oscillatory feedback control schemes is revisited. Here, the general scalar non-linear case is considered, that is,

$$
\dot{x}=f(x)
$$

with $x^{*}$ being an unstable equilibrium point of (1). Let us assume that $f^{\prime}\left(x^{*}\right)=\lambda>0$. Two oscillating feedback control (OFC) schemes will be introduced. The first one, is based on a delayed velocity term as in [6]. In the second method, the perturbation depends on the difference between two delayed terms. Both algorithms will be fully presented in what follows and conditions for stabilization will be deduced. The control objective achievement will be rigorously proven, for these methods applied to the general non-linear case, under the stated conditions. Stability parameters region and control performance of each method when applied to equilibrium point stabilization will be studied and confronted. We will also analyze their possible extensions to the n-dimensional case and to the issue of stabilizing unstable periodic orbits, particularly, in the context of chaos control.

\section{OFC method based on delayed velocity term}

This strategy consists in adding a perturbation based on a delayed velocity term:

$$
\dot{x}(t)=f(x(t))+\epsilon(t) \dot{x}(t-\tau)
$$

where

$$
\epsilon(t)=\left\{\begin{array}{ll}
0, & \text { if } 2 k \tau \leqslant t<(2 k+1) \tau \\
\epsilon, & \text { if }(2 k+1) \tau \leqslant t<(2 k+2) \tau
\end{array} \quad \text { for } k \in \mathbb{N} \cup\{0\} .\right.
$$

being $\epsilon$ and $\tau$ parameters control design.

Let us note that $x^{*}$ is preserved as an equilibrium point of system (2). Then, the objective is to determine $\epsilon$ and $\tau$ so the system may be stabilized in $x^{*}$. Without lost of generality, we can assume $x^{*}=0$ and $f^{\prime}(0)=\lambda$.

When control is activated, $\dot{x}(t-\tau)=f(\varphi(t-\tau))$, being $\varphi$ the solution of (2) in the previous time-interval. Then. system (2) becomes a non-autonomous dynamical system described by a smooth piece-wise ordinary differential equation which has the origin as an equilibrium point. We claim that for a certain range of $\epsilon$, depending on $\lambda$ and $\tau$, it results an asymptotically stable equilibrium point. Therefore, if this strategy is applied with initial condition in a neighborhood of the origin, the control objective is fulfilled. 
The essential ingredients for rigourously proving it are what follow.

Let us call $x(t)$ the solution of (2) with initial condition $x(0)=x_{0}$ and $x_{n}=x(n \tau), n \geqslant 0$. The map $P$ defined by $x_{2 k+2}=P\left(x_{2 k}\right)$ for $k \geqslant 0$ is a key tool in this proof. Then, $x^{*}=0$ is fixed point of $P$ and $P^{\prime}(0)=\widetilde{p}^{\prime}(0) p^{\prime}(0)$ with:

$$
\begin{aligned}
p: x_{2 k+1}=p\left(x_{2 k}\right) & =\phi\left((2 k+1) \tau, x_{2 k}\right) \\
\widetilde{p}: x_{2 k+2}=\widetilde{p}\left(x_{2 k+1}\right) & =\phi\left((2 k+2) \tau, x_{2 k+1}\right)
\end{aligned}
$$

being $\phi\left(t, x_{n}\right)$ the flow associated to the differential equation (2), with initial condition $x_{n}$ and for $t \in[n \tau,(n+1) \tau)$., i.e.,

$$
x(t)=\phi\left(t, x_{n}\right), \quad t \in[n \tau,(n+1) \tau) .
$$

Working out the integral formula of $\phi$ on the respective intervals, it is finally obtained that $p^{\prime}(0)=e^{\lambda \tau}$ and $\widetilde{p}^{\prime}(0)=e^{\lambda \tau}+\epsilon \lambda \tau$. Therefore,

$$
P^{\prime}(0)=e^{\lambda \tau}\left(e^{\lambda \tau}+\epsilon \lambda \tau\right)
$$

which is of modulus less than 1 iff

$$
\frac{-2 \cosh (\lambda \tau)}{\lambda \tau}<\epsilon<\frac{-2 \sinh (\lambda \tau)}{\lambda \tau}
$$

Hence, if $\epsilon$ and $\tau$ verify (4) for $x_{0}$ near the origin, $\lim _{k \rightarrow \infty} x_{2 k}=0$, which implies $\lim _{k \rightarrow \infty} x_{2 k+1}=0$ and it yields to $\lim _{t \rightarrow \infty} x(t)=0$.

Note that if $\epsilon$ verifies (4), $\epsilon=\frac{e^{-\lambda \tau}\left(\alpha-e^{2 \lambda \tau}\right)}{\lambda \tau}$ with $|\alpha|:|\alpha|<1$.

Particulary, if the function is linear, that is, $f(x)=\lambda x$, the map $P$ is also linear, namely, $P(x)=\alpha x$ and the incidence of $\alpha$ on the convergence speed is evident. As an example for the case $f(x)=2 x$, the trajectories resulting from applying (2) with $\tau=0.2$ and $x_{0}=0.5$, $\alpha=-0.4$ and $\alpha=0.8$ are confronted in Figure 1 .
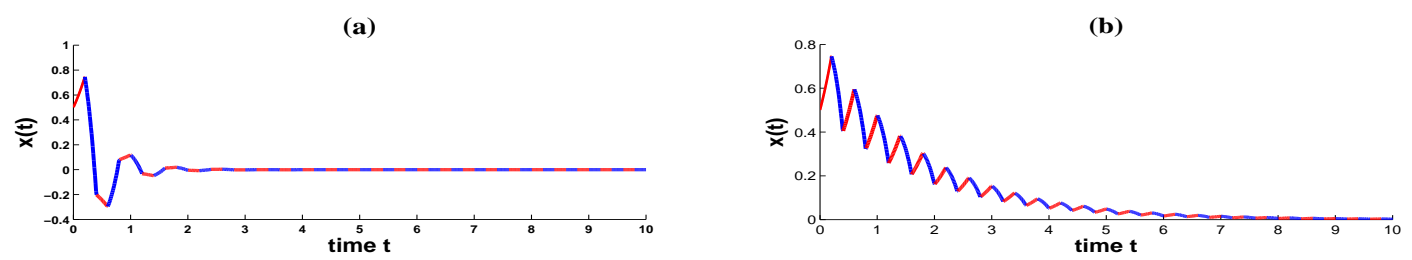

Figure 1: $f(x)=2 x, x_{0}=0.5, \tau=0.2$. (a) $\alpha=-0.4$ (b) $\alpha=0.8$.

On the other hand, the control parameter $\tau$ also affects the convergence of the system trajectories: indeed as $\tau$ is smaller, faster convergence comes out. Taking again $f(x)=2 x$ and $\alpha=-0.4$, and changing $\tau$ by 0.4 , speed of convergence is slower (Figure 2) than in the first example (Figure 1(a)).

Simulations reveal that these effects are inherited by nonlinear case if initial condition is 


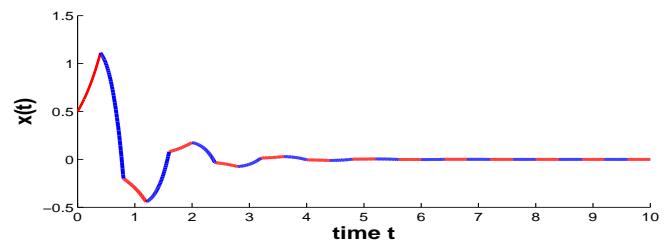

Figure 2: $f(x)=2 x, x_{0}=0.5, \alpha=-0.4, \tau=0.4$.

taken near enough to the origin. This is illustrated for different nonlinear functions, with $\alpha=-0.4, \tau=0.2$ and $x_{0}=0.5$ in Figure 3 (confront to Figure 1(a)).

(a)

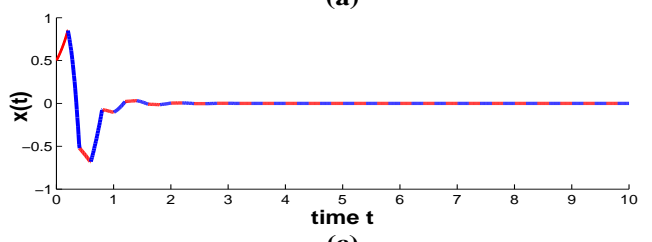

(c)

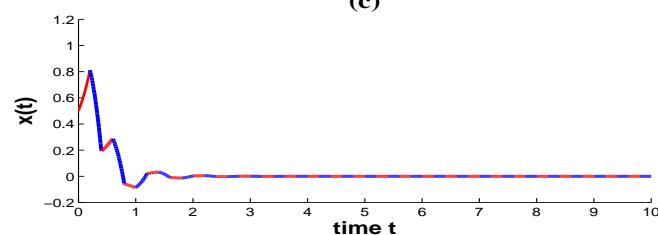

(b)
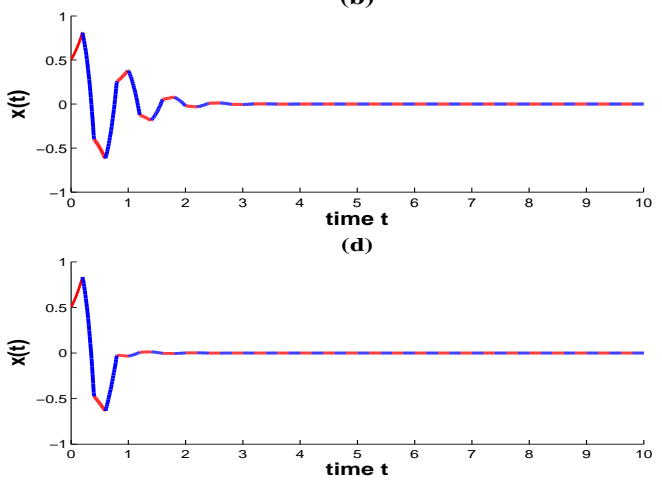

Figure 3: $x_{0}=0.5, \tau=0.2, \alpha=-0.4$. (a) $f(x)=2 x+x^{2}$, (b) $f(x)=2 x+x^{3}$, (c) $f(x)=2 x-x^{3}$, (d) $f(x)=2 x+\sin ^{2}(x)$.

It might be concluded that the rate of convergence is optimized fixing $\alpha$ equal zero and $\tau$ as small as possible. However, choosing $\tau$ too small makes control magnitude take very large values during transitory. In Figure 4(a), the stability parameters region - which is obtained from (4) - is illustrated. Note that if $\tau$ is near zero, for any $\alpha$, there is a dramatic increase of $|\epsilon|$ (Figure 4(b)), so affecting the control performance. Hence, for a given $\alpha$, the choosing of an adequate $\tau$ depends on a compromise between rate of convergence and control magnitude.
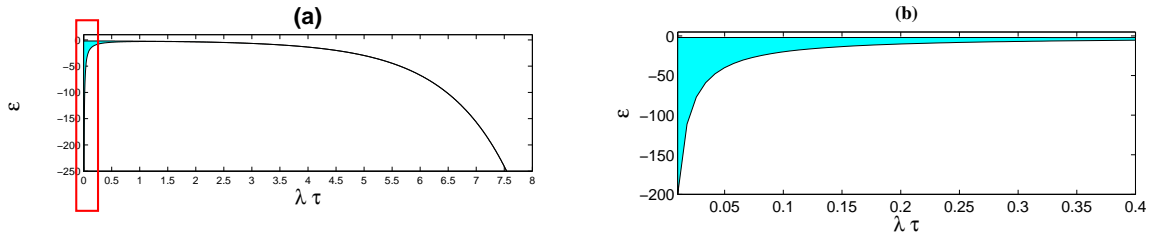

Figure 4: (a) Stability parameters region of (2). (b) Zoom in for $0<\lambda \tau \leqslant 0.4$. 


\section{OFC method based on delayed states difference.}

In this strategy, the perturbation consists in the difference between two delayed states:

$$
\dot{x}(t)=f(x(t))+\epsilon(t)(x(t-2 \tau)-x(t-\tau))
$$

where

$$
\epsilon(t)=\left\{\begin{array}{ll}
0, & \text { if } 3 k \tau \leqslant t<(3 k+2) \tau \\
\epsilon, & \text { if }(3 k+2) \tau \leqslant t<(3 k+3) \tau
\end{array} \quad \text { for } k \in \mathbb{N} \cup\{0\}\right.
$$

As in the first method, $x^{*}$ is preserved as an equilibrium point and without lost of generality, we assume $x^{*}=0$. System (5) also comes out a non-autonomous dynamical smooth piece-wise differential equation and it is also possible to state a range of $\epsilon$, depending on $\lambda$ and $\tau$ such that if this strategy is applied with initial condition in a neighborhood of the origin, the control objective is fulfilled. The proof follows similar steps to the stabilization proof of the first method.

Let $x(t)$ be the solution of (5) with initial condition $x(0)=x_{0}$ and $x_{n}=x(n \tau), n \geqslant 0$. Here, the map $P$ defined by $x_{3 k+3}=P\left(x_{3 k}\right)$ for $k \geqslant 0$, has $x^{*}=0$ as fixed point and $P^{\prime}(0)=\widetilde{p}^{\prime}(0) p^{\prime}(0)$ with:

$$
\begin{aligned}
p: x_{3 k+2}=p\left(x_{3 k}\right) & =\phi\left((3 k+2) \tau, x_{3 k}\right) \\
\widetilde{p}: x_{3 k+2}=\widetilde{p}\left(x_{3 k+2}\right) & =\phi\left((3 k+2) \tau, x_{3 k+2}\right)
\end{aligned}
$$

being $\phi\left(t, x_{n}\right)$ the corresponding flow associated to (5). By using the integral formula of $\phi$ on the respective intervals, it is obtained: $p^{\prime}(0)=e^{2 \lambda \tau}$ and $\widetilde{p}^{\prime}(0)=e^{\lambda \tau}+\epsilon \tau\left(1-e^{\lambda \tau}\right) e^{-\lambda \tau}$. Therefore,

$$
P^{\prime}(0)=e^{3 \lambda \tau}\left[1+\epsilon \tau\left(1-e^{\lambda \tau}\right) e^{-2 \lambda \tau}\right]
$$

which is of modulus less than 1 iff

$$
\frac{e^{3 \lambda \tau}-1}{\tau e^{\lambda \tau}\left(e^{\lambda \tau}-1\right)}<\epsilon<\frac{e^{3 \lambda \tau}+1}{\tau e^{\lambda \tau}\left(e^{\lambda \tau}-1\right)}
$$

or equivalently, $\epsilon=\frac{e^{-\lambda \tau}\left(e^{3 \lambda \tau}-\alpha\right)}{\tau\left(e^{\lambda \tau}-1\right)}$ for $\alpha:|\alpha|<1$.

Comments about the control performance of this method are quite similar to the ones on the first method. For illustration see Figures 5, 6 and 7.
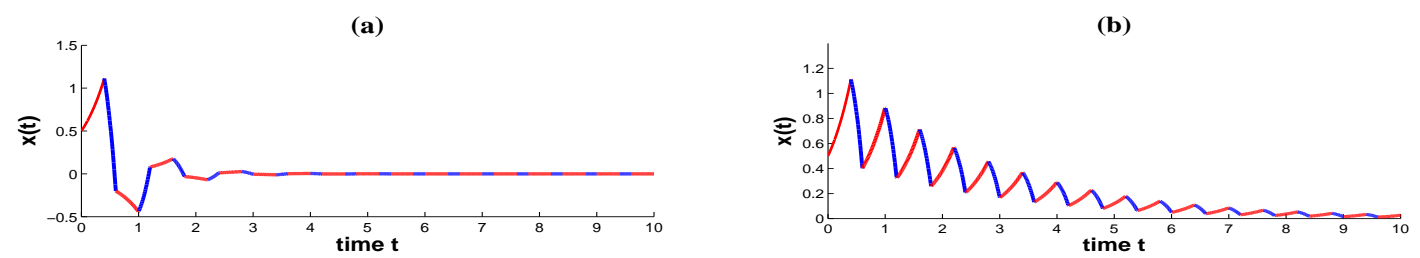

Figure 5: $f(x)=2 x, x_{0}=0.5, \tau=0.2$. (a) $\alpha=-0.4$ (b) $\alpha=0.8$. 


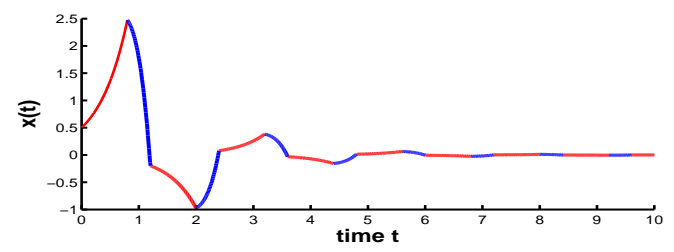

Figure 6: $f(x)=2 x, x_{0}=0.5, \alpha=-0.4, \tau=0.4$.
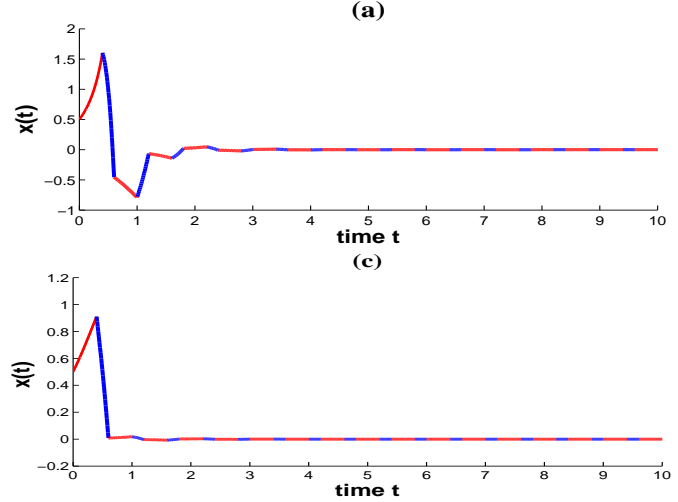

(b)

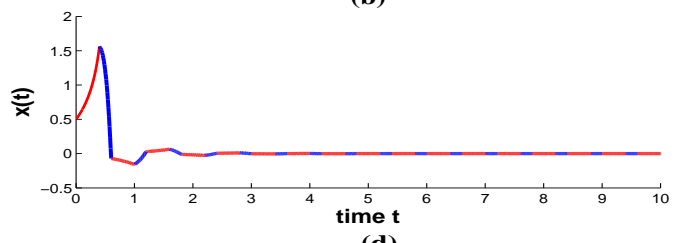

(d)

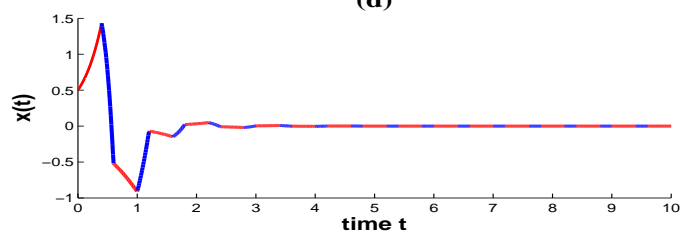

Figure 7: $x_{0}=0.5, \tau=0.2, \alpha=-0.4$. (a) $f(x)=2 x+x^{2}$, (b) $f(x)=2 x+x^{3}$, (c) $f(x)=2 x-x^{3}$, (d) $f(x)=2 x+\sin ^{2}(x)$.

Equation (7) states the stability parameters region of this method. Graphically, it is seen in Figure 8:
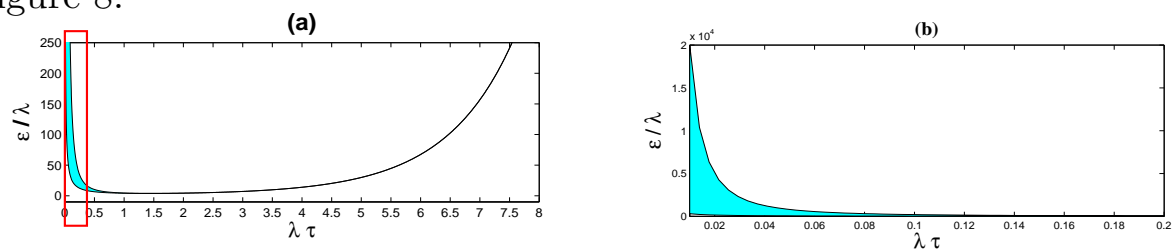

Figure 8: (a) Stability parameters region of (5). (b) Zoom in for $0<\lambda \tau \leq 0.2$.

Considerations about the choosing of the design control parameters are similar to the ones of the first introduced method.

\section{Concluding remarks and future research}

Many simulated examples let us conjecture that the first introduced method displays better control performance features than the second one. This may even be noted in the few cases shown in this work, (compare Figures of Section 2 with Figures of Section 3 for the corresponding cases).

On the other hand, the rate of convergence may be quantified (by obtaining an exponential bound of the solution). Based on this index of convergence and on a detailed analysis of 
the stability parameter region of each method, the conjectured results are confirmed.

However, the second method must not be discarded at all. Firstly, because sometimes it may be relevant to avoid the computation of derivatives. The second reason has to do with stabilization of unstable periodic orbits (UPO). Let us say that the methods and results developed in this work may be straight forwards transferred to the n-dimensional case. Now suppose that $\widetilde{x}(t)$ is a UPO and its period $T$ is known. By introducing $\delta x=x-\widetilde{x}(t)$, the problem of UPO stabilization is re-formulated as the problem of stabilizing the origin in the n-dimensional case.

The oscillating feedback control based on delayed states becomes:

$$
u(t)=K(t)[\delta x(t-2 T)-\delta x(t-T)]=K(t)[x(t-2 T)-x(t-T)]
$$

being $K(t)$ the oscillating control gain. As in Pyragas method, it does not require the exact location of the UPO to be stabilized. This proposal, and namely, its application for controlling chaos, i.e., for the UPO embedded in a strange attractor, is part of our future research.

\section{Acknowledgments}

This work is partially supported by CONICET PIP (KE2) 2012-2014 and Programación UBACyT 2014-2017 GEF.

\section{References}

[1] O. Morgul, On the stabilization of periodic orbits for discrete time chaotic systems, Physics Letters A, vol. 335, 127-138, (2005).

[2] H. Nakajima and Y. Ueda, Limitation of generalized delayed feedback control, Physica D, vol. 111, 143-150, (1998).

[3] K. Pyragas, Continuous control of chaos by self-controlling feedback, Phys. Lett. A., 421-428, (1992).

[4] K. Pyragas, Control of chaos via extended delay feedback, Phys. Lett. A, vol. 206, 323-330, (1995).

[5] K. Pyragas, Control of chaos via an unstable delayed feedback controller, Physical Review Letters, vol. 86, (2001).

[6] H. G. Schuster and M. B. Stemmler, Control of chaos by oscillating feedback, Physical Review E, vol. 56 N6, 6410-6416, (1997).

[7] J. E. S. Socolar and D. W. Sukow and D. J. Gauthier, Stabilizing unstable periodic orbits in fast dynamical systems. Phys. Rev.E 50, 3245-3248, (1994). 